\title{
Politique
}

Politique

\section{L'analyse politique critique au Canada anglais et la question du Québec, 1970-1993}

\section{Serge Denis}

Numéro 23, hiver 1993

Tendances de la science politique au Québec

URI : https://id.erudit.org/iderudit/040751ar

DOI : https://doi.org/10.7202/040751ar

Aller au sommaire du numéro

\section{Éditeur(s)}

Société québécoise de science politique

ISSN

0711-608X (imprimé)

1918-6584 (numérique)

Découvrir la revue

Citer cet article

Denis, S. (1993). L'analyse politique critique au Canada anglais et la question du Québec, 1970-1993. Politique, (23), 171-209. https://doi.org/10.7202/040751ar
Résumé de l'article

Il y aurait beaucoup à dire sur les théories élaborées au Canada anglais sur les réalités québécoises depuis le $\mathrm{XIX}^{\mathrm{e}}$ siècle. Nous verrons de quelle façon le nationalisme québécois et ses revendications constitutionnelles ont été analysées dans les universités canadiennes, durant les vingt-cinq dernières années. Il est possible de retracer chronologiquement l'hégémonie de quelques grands paradigmes théoriques, qui se suivent selon des enchaînements à la fois de filiation et de rupture. L'attachement aux institutions étatiques tend pourtant à apparaître comme une constante desdits paradigmes, conditionnant dans certains cas la perception des phénomènes circonscrits et, ce qui devient paradoxal, la définition démocratique même des rapports entre populations et... institutions. 


\title{
L'ANALYSE POLITIQUE CRITIQUE AU CANADA ANGLAIS ET LA QUESTION DU QUÉBEC, 1970-1993 *.
}

\author{
Serge Denis
}

Université d'Ottawa

II y aurait beaucoup à dire sur les théories élaborées au Canada anglais sur les réalités québécoises depuis le $\mathrm{XIX}$ • siècle. Nous verrons de quelle façon le nationalisme québécois et ses revendications constitutionnelles ont été analysées dans les universités canadiennes, durant les vingt-cinq dernières années. Il est possible de retracer chronologiquement l'hégémonie de quelques grands paradigmes théoriques, qui se suivent selon des enchaînements a la fois de filiation et de rupture. L'attachement aux institutions étatiques tend pourtant à apparaître comme une constante desdits paradigmes, conditionnant dans certains cas la perception des phénomènes circonscrits et, ce qui devient paradoxal, la définition démocratique même des rapports entre populations et... institutions.

Les développements souvent tumultueux de la vie politique canadienne au cours des dernières décennies ont contribué à décupler l'attention que les sciences sociales, en

- Cet article résulte d'une mise à jour de la communication présentée au colloque de la Société québécoise de science politique, tenu à l'Université Laval dans le cadre du congrès de l'Acfas, en mai 1990. La communication a servi à la rédaction du livre Le long malentendu, publié aux éditions du Boréal en 1992, dans lequel on retrouve certains des points de vue exposés ici. Je remercie les évaluateurs anonymes de la Revue pour leurs conseils judicieux.

- Dans le présent article, le générique masculin est utilisé sans aucune discrimination et uniquement dans le but d'alléger le texte.

Revue québécoise de science politique, no 23, hiver 1993. 
particulier au Canada anglais, ont porté traditionnellement à l'évolution des rapports constitutionnels et à la situation des minorités ethniques et linguistiques au pays. Outre les avancées évidentes des études en économie politique, il est manifeste que la volonté de cerner la réalité du Québec contemporain, notamment, a ébranlé certains schèmes par lesquels étaient couramment abordées jusque-là les grandes questions de gouvernement.

II nous a semblé utile, en cette période où les discussions sur l'avenir de la Confédération sont en suspens, de revenir à l'analyse de la question québécoise, telle qu'elle a été conduite et s'est modifiée au Canada anglais depuis environ un quart de siècle. Évidemment, le cadre d'un article ne permet pas d'être exhaustif. Nous avons donc décidé de nous en tenir, ici, à la présentation des paradigmes élaborés par l'analyse politique critique.

Nous employons l'expression ranalyse politique critique" dans son sens le plus large, ce qui, nous en convenons, peut prêter à contestation. Mais nous voulons signaler par là que nous nous arrêterons prioritairement aux tendances théoriques qui se réclament (plus explicitement que ce n'est le cas des apports traditionnels des sciences politiques) de principes de progrès social et démocratique, de justice redistributive aussi, et qui fondent leur évaluation de l'État canadien sur ces mêmes principes. Nous privilégierons les œuvres produites par des intellectuels anglophones vivant à l'extérieur du Québec et dont les travaux ne s'inscrivent pas au compte d'une participation militante à l'élaboration politique de courants de gauche organisés (NPD, PC, maoïstes ou trotskystes). Ce n'est pas que nous discréditons les analyses issues de ces milieux', mais leur appréciation

1. Par exemple, nous tenons le livre de Stanley B. Ryerson, Le capitalisme et la Confédération, Montréal, Parti Pris, 1972, $1^{10}$ édition, pour un apport scientifique décisif. D'un autre cóté, nous pensons que Henry Milner, *The Anglophone Left in Québec and National Self-Determination*, in Caldwell, G. et E. Waddell (dir.), The English of Québec, Québec, IORC, 1982 , p. 399-414, a exposé les hypothèses interprétatives rendant compte de la situation des progressistes anglophones du Québec durant la période que nous étudions. 
supposerait de se pencher sur des facteurs historiques, politiques et sociologiques que nous ne pouvons ici traiter.

Nous grouperons sous quelques thèmes principaux les idées-forces les plus représentatives des grandes interprétations de la question du Québec et du mouvement national québécois. Pour mieux illustrer les éléments de continuité et de césure entre ces interprétations, l'exposition ne suivra pas un ordre chronologique : nous partirons plutôt d'une présentation des axes d'analyse qui paraissent hégémoniques à l'époque du référendum du Parti québécois pour les comparer ensuite aux grandes orientations du début des années 1970, et cerner enfin à l'occasion des débats sur l'Accord du lac Meech et le référendum de l'automne 1992 leurs transmutations ou leurs conséquences récentes. Nous ne visons pas, en citant certaines œuvres, à stigmatiser des auteurs, mais nous voulons indiquer des contributions particulièrement influentes.

\section{Fédéralisme et progrès social}

Faisant le bilan des apports théoriques du nationalisme canadien-anglais entre 1964 et 1984, Daniel Drache écrivait, il y a quelques années, que le caractère progressiste des tendances visant l'indépendance économique face aux ÉtatsUnis était continuellement «miné par la position profondément réactionnaire du Canada anglais sur le Québec". S'arrêtant plus spécifiquement au nationalisme de gauche, dans lequel se reconnaissent la majorité des auteurs qui pratiquent ce que nous avons appelé l'analyse politique critique, Drache décelait deux courants intellectuels principaux : celui qu'il identifiait à Garth Stevenson, critique sévère du $P O$ et opposant déclaré au séparatisme, et celui de Reg Whitaker, qu'il ne qualifiait pas. Selon lui, les deux décennies qu'il évaluait n'avaient donné lieu au Canada anglais à aucune analyse nouvelle de quelque intérêt sur la place du Québec au sein de la 
Confédération, après l'œuvre pionnière de Stanley B. Ryerson ${ }^{2}$.

Dans l'introduction à son importante étude sur le Québec contemporain, William D. Coleman souligne que, "malgré la viabilité continue de son mouvement" en faveur de I'indépendance, la "compréhension (qu'en a le Canada anglais) demeure pauvre ${ }^{3} n$. Nous allons d'abord tenter de cerner les traits principaux de cette "compréhension" du Québec, telle qu'elle se manifestait au tournant de la décennie 1980, en partant des pistes indiquées par Drache.

La problématique du couple "centralisation-fragmentation"

L'une des constantes de l'analyse de gauche au Canada anglais sur les problèmes constitutionnels et la question du Québec relève du prisme de l'opposition centralisationfragmentation : on le sait, les volontés de décentralisation (qui conduiraient à la fragmentation) du fédéralisme canadien y sont expliquées par l'attraction qu'exerce sur les diverses parties du pays l'intégration continentale sous domination américaine. Le professeur Garth Stevenson, spécialiste des systèmes fédéraux et en particulier du fédéralisme canadien, a le plus systématiquement élaboré ce point de vue.

Déjà, à la mi-décennie 1970, il avait cherché à rendre compte des développements du nationalisme québécois depuis la Révolution tranquille par un unique facteur déterminant : «le néo-nationalisme de la petite-bourgeoisie francophone". Durant tout le $X X^{\bullet}$ siècle, les relations économiques nord-sud entre chacune des régions du Canada et les États-Unis se sont accentuées, écrivait-il, ce qui a entraîné l'élargissement des pouvoirs provinciaux au détriment du gouvernement central. Pour des raisons historiques

2. Daniel Drache, *Whatever Happened to Canadian Nationalism», in Canadian Dimension, vol. 18, no 5, oct.-nov. 1984, p. 1520.

3. William D. Coleman, The Independence Movement in Québec, 1945-1980, Toronto, University of Toronto Press, 1984, p. 4. 
particulières, cette tangente est portée au Québec par la petite-bourgeoisie et son projet "néo-nationaliste ${ }^{4}$.

Vers la fin de la même décennie, Stevenson a articulé plus complètement cette analyse et achevé sa formulation ${ }^{5}$. Dans son livre Unfulfilled Union, il caractérise les points de vue prônant la décentralisation de «centrifuges" et souligne que leur renforcement incessant menaçait alors de conduire à une situation où la structure étatique fédérale *deviendrait totalement impropre à quelque utilisation ${ }^{\circ} x$. Revenant sur I'histoire constitutionnelle du Canada, il montre qu'on ne peut trouver l'origine profonde des grandes périodes de centralisation et de décentralisation dans des facteurs d'ordre politique, juridique ou intellectuel. Les causes fondamentales, il les repère dans l'évolution de l'économie canadienne et ce qu'il appelle le kprovince-building", l'effort conscient d'extension des champs de juridiction et de renforcement de l'appareil administratif des provinces, qu'il relie à la première cause.

4. Garth Stevenson, «Federalism and the Political Economy of the Canadian Statex, in Leo Panitch, dir., The Canadian State, Toronto, University of Toronto Press, 1977.

5. Nous nous référons essentiellement à : i) Garth Stevenson, Unfulfilled Union, Toronto, Macmillan, 1979; ii) Garth Stevenson, «The Division of Powersw, in Richard Simeon, dir., Division of Powers and Public Policy, vol. 61, Commission Macdonald, Ottawa, 1985; iii) Garth Stevenson, "Canadian Regionalism in Continental Perspective», Journal of Canadian Studies, no 15, 1980. Ces deux derniers articles ont été réédités in : Olling, R.D. et M.W. Westmacott, dir., Perspectives on Canadian Federalism, Scarborough, Prentice-Hall Canada Inc., 1988. II faut par ailleurs noter que la $3^{*}$ édition de son livre Unfulfilled Union (Gage Education Publishing Co., 1989), devenu un classique, montre une évolution importante du point de vue de l'auteur sur le Québec. Ainsi qu'il l'indique dans sa préface, «le chapitre sur le nationalisme du Québec a été presque entièrement récrit" ; il $n^{\prime} y$ reprend plus cette hypothèse, par exemple, selon laquelle le nationalisme québécois serait identifiable à un simple courant d'intégration économique continentale. En nous servant de ses écrits antérieurs, nous visons à illustrer l'une des interprétations marquantes du mouvement national québécois au tournant de la décennie 1980.

6. Garth Stevenson, Unfulfilled Union, op. cit., 1979, p. 76. 
Les développements de l'économie canadienne ont donné naissance, explique-t-il, à de puissants intérêts régionaux qui soulèvent du mécontentement devant les politiques prétendant s'adresser aux besoins de cette économie prise comme un tout. Comme, d'un autre côté, les pouvoirs publics au Canada ont traditionnellement participé à la vie économique, plus que ceux $d$ 'autres pays occidentaux, les différences entre les intérêts des régions se sont naturellement manifestées dans les orientations des gouvernements provinciaux, ce qui a entraîné le phénomène du «province-building". Depuis la fin de la Seconde Guerre, ces processus ont été accélérés, car il y a eu baisse relative, en plus, du poids des secteurs économiques capables de porter l'affermissement de l'autorité du gouvernement central (par exemple, «les banques», «les compagnies maritimes et les chemins de fer transcontinentaux ${ }^{7}$ ).

Or, ainsi que Stevenson l'a à nouveau exprimé plus récemment, une politique de "nationalisme économique» ne peut s'appuyer que sur «un gouvernement central fort". Le Canada ayant été formé "en dépit (des lois) du marché», s'en remettre au seul jeu de ces lois revient à prôner «l'intégration continentale" dans l'empire économique américain ${ }^{8}$. Pour éviter cette éventualité, il faut s'arc-bouter à une stratégie de nationalisme économique, qui requiert la nette prédominance d'Ottawa sur les législatures provinciales.

Ce n'est pas que Stevenson identifie péremptoirement le gouvernement central aux politiques de progrès, mais il juge le renforcement du pouvoir fédéral nécessaire à la résistance économique à l'emprise américaine, et pose toutes les oppositions à ce renforcement, pêle-mêle, comme relevant de tendances à la continentalisation. Les aspirations nationales du Québec pesaient donc dans le sens de la réaction politique. Elles s'expliquaient essentiellement, depuis les années 1960, comme projet politique d'une petite-bourgeoisie qui voulait

7. Ibid., p. 91.

8. Garth Stevenson, \&The Agreement and the Dynamics of Canadian Federalism*, in Gold, Marc et David Leyton-Brown, dir., Trade Offs on Free Trade, Toronto, Carswell, 1988, citation p. 138. 
élargir ses liens économiques avec les États-Unis. Stevenson, homme de gauche, voyait donc le nationalisme québécois avec hostilité, dénonçant en 1979 «qu'un gouvernement provincial propose de briser la fédération sur la base d'un référendum confinéw aux frontières de son territoire, quand on trouve abusif et killégitime (...) d'amender la constitution sur la base d'un référendum auquel tous les Canadiens pourraient participer"

\section{La nature politique de l'État canadien}

D'autres analystes s'étaient employés, depuis le milieu des années 1970, à cerner plus directement les rapports politiques et idéologiques qui rendent compte de la nature du Canada et de I'histoire de son fédéralisme. À cet égard, la contribution de Reg Whitaker est en effet intéressante parce qu'elle témoigne, au fil des ans, d'un effort pour développer l'étude critique à la fois de l'État canadien et du nationalisme québécois. De plus, les points de vue de l'auteur semblent correspondre à un état d'esprit très répandu chez les universitaires progressistes anglophones dans la seconde moitié des années 1970 : attirés pendant une époque par l'élan du nationalisme québécois, ils cherchent à donner droit a ses revendications, mais restent craintifs devant ses conséquences ultimes pour l'État canadien.

Whitaker s'est d'abord signalé en mettant l'accent sur la faiblesse des traditions démocratiques du Canada, sur la faible part qu'ont tenue les principes de la démocratie dans la mise en forme de ses institutions ${ }^{10}$. II a établi un lien de

9. Garth Stevenson, Unfulfilled Union, op. cit., 1979 , voir les pages 217-218.

10. Pour notre présentation de la pensée de Whitaker sur la question nationale et les problèmes constitutionnels, nous nous référons principalement à ses études : i) «Images of the State in Canadan, chapitre 2 de : Panitch, Leo, dir., The Canadian Stage, op. cit., ii) «Democracy and the Canadian Constitutionw, chapitre 12 de : Banting, K. et R. Simeon, dir., And No One Cheered, Toronto, Methuen, 1983; iii) «The Québec Cauldron, a Recent Account», chapitre 6 de : Gagnon, Alain G., dir., Québec : State and Society, Toronto, Methuen, 1984. 
continuité rectiligne entre ces antécédents et les processus d'adoption des nouveaux documents constitutionnels durant les années 1980, décriés comme non démocratiques parce que ne reposant sur aucune participation populaire décisive. En une formule-choc, il a raillé la négociation qui a conduit au rapatriement de l'Acte de l'Amérique du Nord britannique (AANB) et à la promulgation de la Loi constitutionnelle du Canada en 1982 : le pays, a-t-il écrit, n'est pas une «communauté de communautés, comme disait Joe Clark, mais une communauté de gouvernements qui ne pouvaient s'entendre que sur une seule chose, garder le peuple hors des discussions" ".

II est évident, explique Whitaker, que dans chaque système fédéral la légitimité doit s'exprimer et se façonner sur le terrain de plusieurs niveaux de gouvernement. Le problème du Canada, c'est que les règles de la démocratie n'ayant pas présidé à sa naissance, les ententes sur l'aménagement interne de la fédération ont toujours reposé uniquement sur les marchandages entre élites et sur les diverses instances étatiques. Cette histoire fragilise politiquement l'existence de la "société fédérale" et l'attachement populaire à son endroit (il y a eu absence de phénomènes de légitimation) ${ }^{12}$. II a opposé, en ce sens, aux processus de négociation constitutionnelle qui ont prévalu en 1981-1982, l'idée de référendums pancanadiens, faisant notamment référence au projet de Pierre E. Trudeau à cet égard. De tels référendums conforteraient la légitimité de la "société fédérale» et démocratiseraient les démarches de renouvellement de la constitution.

Deux brèves remarques à ce sujet. D'abord, il paraît bien difficile de voir en quoi la tenue d'un référendum pancanadien, dans la conjoncture du tournant de la décennie 1980, aurait permis de rompre avec les vieux moules constitutionnels non démocratiques. Face au Québec, et cela a valeur aussi pour les peuples autochtones, ce type de

11. Reg Whitaker, «The Trudeau Era», Canadian Dimension, vol. 18, no 5, oct.-nov. 1984, p. 9.

12. Voir *Images of the State...», op. cit., p. 46-47. 
référendum, remplaçant les négociations avec la province ou consignant des formules qu'aucun groupe québécois représentatif $n$ 'appuyait, serait plutôt apparu comme un déni brutal de l'autodétermination. En fonction même des réalités canadiennes contemporaines, pour être compatible avec les principes de la démocratie, il aurait fallu qu'un éventuel référendum de cette nature se réalise sur la base d'un document préalablement négocié et accepté par les diverses composantes de la population du pays, chacune d'elles ayant élaboré ses positions en assurant la plus grande participation populaire. Dans le cas contraire, non seulement les minorités ethniques et linguistiques se seraient-elles senties bousculées politiquement, mais on voit mal comment la «société fédérale" aurait été renforcée : le risque eût plutôt été grand d'une accentuation des traits du fédéralisme canadien qui en sont venus à expliquer la faiblesse de l'identification à l'État central.

Cette considération entraîne notre deuxième remarque. De manière peut-être étonnante, il semble qu'il faille relier l'absence théorique de cette étape (d'une adoption préalable par les populations des documents constitutionnels qui pourraient être soumis à un référendum pancanadien) à I'analyse même que donne Whitaker des développements de la pensée politique et des institutions dans le HautCanada/Canada-Ouest au XIX ${ }^{\bullet}$ siècle $^{13}$. II y caractérise correctement, pensons-nous, les principaux éléments qu'il identifie, mais il ne prend pas la juste mesure de ce qui a été vaincu avec les troupes de William Lyon Mackenzie : très directement, il s'agissait des principes de la définition démocratique de la souveraineté et de la légitimité politique, comme de leur corollaire en situation coloniale qu'est l'exercice de l'autodétermination. Sur cette question, il faut voir que les droits d'aucune des populations des territoires britanniques d'Amérique du Nord n'ont pu affirmer leur

13. Voir *lmages of the State...», ibid., et son «Democracy and the Canadian Constitution*, op. cit.; notre jugement s'inspire ici de l'essai sur les origines de la Confédération intitulé "La fondation de l'État au Canada*, p. 15-51 in Roch Denis, (dir.), Luttes de classes et question nationale au Québec, 1948-1968, Montréal, PSI, Paris, EDI, 1979. 
prééminence, même pas ceux de leur fraction historique d'origine européenne anglo-saxonne. $D^{\prime}$ où cette matrice constitutionnelle confirmée par l'Acte d'Union, et sur la base de laquelle on se dirigea vers l'AANB de 1867 : ultimement, la souveraineté politique procédait au Canada du sommet de la société plutôt que de sa base. L'addition, en 1981-1982, d'un référendum à un processus de réaménagement constitutionnel à nouveau défini par le haut (le cabinet fédéral) n'aurait pas permis, du point de vue de la démocratie, de faire disparaître cette caractéristique de document octroyé (donc placé, à tout le moins partiellement, sous le contrôle des instances qui octroient) qu'ont toujours manifestée les lois organiques du Canada, y compris pour le groupe linguistique majoritaire.

Philip Resnick est revenu, au tournant de la décennie 1990, à cette problématique des traits non démocratiques de l'expérience politique canadienne. II a cherché à la baliser en partant du modèle théorique des «constitutions mixtes» et des conceptions idéologiques burkéennes qui ont inspiré l'évolution du système étatique de la Grande-Bretagne, dont il fait découler le mode de démarches constitutionnelles au Canada, qui systématiquement procèdent du haut vers le bas («top-down version of constitution-making") ${ }^{14}$. Éléments d'interprétation qui, très certainement, contribuent à la conceptualisation de dimensions véritables des réalités canadiennes.

Mais ce qui est paradoxal dans les études soulignant la faiblesse et les failles des assises démocratiques du Canada, c'est que personne, réellement, (mais s'il y a erreur, elle indique aujourd'hui une exception qui confirme la règle) ne poursuit l'analyse jusqu'à l'institution qui explicitement les condense et les porte : la monarchie britannico-canadienne, la perpétuation des structures d'un régime monarchique en cette fin même du $X X^{\circ}$ siècle. La version "top-down" de la légitimité politique, qui inclut le sens des démarches ayant instauré et instaurant la communauté canadienne, tout comme

14. Philip Resnick, The Masks of Proteus : Canadian Reflections on the State, Montréal et Kingston, McGill-Queen's University Press, 1990; p. 74-82, chapitre 5 et p. 149 pour la citation. 
les caractéristiques non démocratiques des règles y définissant les contours de la souveraineté, trouvent leur symbole et leur vérité dans la monarchie maintenue. Tout le monde aura compris qu'il ne s'agit pas de prétendre qu'Elisabeth II est partie prenante des décisions du cabinet fédéral, ni même qu'elle les vérifie. Mais les formes de la monarchie constitutionnelle composent l'architecture du système institutionnel du Canada, dans laquelle ont été et sont imbriquées les limites démocratiques de ce système.

Ce qui ne cesse d'étonner, c'est qu'en traitant des fondements idéologiques et politiques de l'État et de la vie constitutionnelle au Canada, les progressistes anglophones ne $s^{\prime}$ arrêtent pas à l'existence de cette monarchie, même quand ils disent se situer sur le terrain des principes de la démocratie. Ne tenant pas compte d'un pan important de la réalité, les points de vue sont alors scientifiquement inachevés. II ne serait pas juste, pensons-nous, de relier ce creux à un attachement héréditaire du Canada anglais, pris comme un monolithe, à la royauté britannique. Plus fondamentalement, il provient de la difficulté à poser les caractéristiques critiquées comme éléments constitutifs majeurs du système politique, c'est-à-dire à percevoir que l'ensemble ainsi conjugué procède structurellement de ces caractéristiques, dont la monarchie est le couronnement.

Cela étant, pour Reg Whitaker, les griefs historiques des francophones sont légitimes, et il a mis en relief certaines inégalités de traitement entre francophones et anglophones inscrites dans l'AANB lui-même. Mais ces griefs ne semblent pas, chez lui, procéder de traits historiques et institutionnels centraux de l'État canadien. De sorte qu'il a pu expliquer le nationalisme de la Révolution tranquille comme "l'idéologie de la nouvelle classe moyenne qui exprimait ses revendications d'une place au soleil en un langage qui, sans aucun doute, était honnêtement nationaliste dans l'identité culturelle qu'il se donnait, mais qui, en même temps, représentait une expression de son intérêt de classe, ou plus précisément de fraction de classe ${ }^{15} "$. Notons bien : il ne parle pas de

15. Reg Whitaker, «The Québec Cauldron», op. cit., p. 76. 
l'expression idéologique d'un mouvement d'autodétermination qui remettrait en cause des caractéristiques constitutives non démocratiques de la Confédération, mais il mentionne l'expression des intérêts d'une «fraction de classex. Sans nier l'existence de ce type d'éléments, ils nous paraissent ici conjoncturels, ne pouvant rendre compte ni du caractère récurrent de la question nationale québécoise ni de sa qualité de mouvement populaire, qui définissait aussi le nationalisme depuis la Révolution tranquille.

Contrairement à Garth Stevenson, Whitaker n'a pas analysé, en règle générale, le nationalisme québécois contemporain comme un courant d'essence réactionnaire. Mais la dissociation qui est finalement opérée entre sa dynamique et la nature, bien réactionnaire quant à elle, de certains fondements de l'État canadien lui fait malgré tout craindre ses conséquences les plus ultimes. Avec le sens de la formule qui est le sien, il a ainsi écrit que, "l'ironie tragique», pour "les socialistes canadiens-anglais», était précisément que «même une victoire partielle du progressisme au Québec (qui se reconnaissait dans l'indépendantisme) risquait d'être gagnée au prix d'un démembrement de la nation canadienne ${ }^{16}$ ", accentuant la menace d'une intégration au géant américain. Pris en soi, le nationalisme québécois de la dernière période n'était donc pas droitier, mais il faisait peser sur l'ensemble des parties du Canada de bien sombres perspectives.

II est intéressant de signaler que, pour la dernière quinzaine d'années, et même en remontant quelque peu audelà, cette manière de concevoir le mouvement national au Québec est devenue un véritable paradigme. Non pas que les théries au Canada anglais disent toutes la même chose, mais elles partagent cette disposition fondamentale à vouloir faire correspondre le plus étroitement possible les aspirations nationales aux revendications propres d'une classe-support, de nature bourgeoise. Contentons-nous d'illustrer brièvement ce trait à partir des explications contenues dans deux études sur le Québec que nous considérons comme des contributions

16. Reg Whitaker, elmages of the Staten, op. cit., p. 66. 
majeures : celle de McRoberts-Posgate et celle de Coleman"1.

Ainsi, Kenneth McRoberts et Dale Posgate, qui ne craignent pas de parler couramment de knation québécoise", semblent eux aussi percevoir l'éclosion de son nationalisme récent dans l'affirmation politique d'une «nouvelle classe moyenne*, qu'ils retracent prioritairement chez ceux qu'ils nomment (avec d'autres) les «travailleurs du langage». Ils considèrent la Loi 101 comme une illustration, à la fois, de la "modération» péquiste et des objectifs spécifiques de ces "travailleurs du langage", au premier chef d'une fraction d'entre eux : "les enseignants francophones", qui "éprou(vaient) une vive inquiétude devant le nombre décroissant des nouveaux élèves" dans le secteur scolaire de langue française. On voulait également assurer un rôle plus grand à «la langue française et aux francophones dans l'économie" ${ }^{18}$.

Plus récemment, William $D$. Coleman a repris avec minutie l'analyse du mouvement indépendantiste québécois depuis 1945, en réévaluant spécifiquement plusieurs des grandes hypothèses explicatives qu'on en a données ${ }^{19}$. II a cherché à dépasser les cadres interprétatifs restreints et est arrivé à une explication globale de nature sociologique. Selon lui, avec la réduction du poids et de l'influence des institutions qui avaient servi jusqu'aux années 1960 à définir la société francophone (homogénéité confessionnelle, organisation

17. Kenneth McRoberts et Dale Posgate, Développement et modernisation du Québec, Montréal, Boréal, 1983; William D. Coleman, The Independence Movement in Québec, 1945-1980, op. cit.

18. Kenneth McRoberts et Dale Posgate, op. cit., p. 229-230. Cette notion d'un rôle décisif que tiendraient les «travailleurs du langage* dans un mouvement nationaliste semble découler, à notre connaissance, des vieux points de vue de l'extrême-gauche de la $2^{\circ}$ Internationale lors des discussions d'avant la Première Guerre sur le problème des nationalités, notamment dans l'empire austro-hongrois : voir, par exemple, les textes d'Otto Strasser et Anton Pannekoeck réunis par Claudie Weill sous le titre Nation et lutte de classe, 10/18, série «La nation en question», Paris, 1977, où cette notion de «travailleurs du langage» est directement utilisée.

19. William D. Coleman, op. cit., voir notamment les pages 5-15. 
traditionnelle du système éducatif et des services sociaux...), une crise profonde d'identité a secoué la province. Seule la langue restait le facteur distinctif commun, d'où l'importance des luttes linguistiques; mais séparée des anciennes valeurs dominantes, elle ne pouvait combler tous les besoins d'identité collective.

Trois groupes principaux ont alors cherché à redonner un sens à la destinée nationale, dont la coalition est à la base du mouvement indépendantiste apparu depuis la Révolution tranquille. D'abord, le mouvement syndical, selon lequel la souveraineté politique devait permettre un contrôle et une planification de l'économie; puis, l'intelligentsia qui voulait garantir par la souveraineté le caractère culturel distinct du Québec, gage du maintien de ses liens propres avec la population; enfin, les «classes moyennes" («professions traditionnelles, cols blancs du secteur public, enseignants») qui avaient perdu, avec la dissolution des anciens systèmes d'éducation et de services sociaux, la place de leurs prédécesseurs dans la vie collective et qui voulaient la reconquérir par la mise au point d'un projet national global ${ }^{20}$.

L'analyse de Coleman ne situait pas dans la dimension constitutionnelle pancanadienne la question du Québec, et I'interprétation qu'il proposait de la coalition indépendantiste est une construction partiellement composite, conjuguant notamment mouvements structurés et courants d'idées. Elle illustrait néanmoins avec force la tentative de sortir des schèmes cernant par l'existence d'un secteur socioéconomique limité la nature du nationalisme québécois, tout comme - contradictoirement - elle montrait le caractère paradigmatique de ce type d'analyse, puisqu'elle aboutissait malgré tout à une explication construite par la juxtaposition des mouvements de tels secteurs.

En revenant à l'essence de notre propos initial, il faut souligner que deux traits principaux balisent donc les études produites par les intellectuels anglophones progressistes sur le Québec, de la seconde moitié des années 1970 au début de la décennie suivante. Que ce soit en fonction de

20. Ibid., p. 15-19, et chap. 8, p. 224 sqq. 
préoccupations d'ordre surtout économique-social, d'ordre politique ou d'ordre culturel, il y a une crainte que l'effet ultime des demandes du Québec, et singulièrement l'exercice de l'autodétermination, résulte en une absorption des diverses régions du Canada par les États-Unis. Ensuite, il y a une compréhension incomplète (c'est-à-dire non achevée) du mouvement national de masse au Québec, qui est liée à la caractérisation inachevée du type de rapports entre les populations et entre ces populations et l'État, consignés par I'AANB. Cela posé, la peur d'une «balkanisation" du fédéralisme canadien a conduit une partie de cette gauche à voir le salut progressiste du pays dans un raidissement de l'État central. $\dot{A}$ ce stade, elle en fut plongée dans un véritable dilemme parce que ses principes politiques la conduisaient aussi à être sensible au droit du Québec à décider librement de son avenir. Contrairement à une légende tenace, il n'est pas vrai que l'intelligentsia progressiste du Canada anglais se serait signalée alors par un appui quasi unanime au nationalisme québécois et une identification à sa cause. A l'époque du référendum de 1980, elle tendait à se disposer plutôt sur des axes diversifiés, nés précisément de l'impact de son dilemme politique ${ }^{21}$.

\section{Une rupture avec l'héritage de la période "waffle»?}

La gauche intellectuelle, dont nous venons de rappeler les thèses, inscrivait ses analyses dans l'effort de renouvellement des explications de la réalité canadienne, et notamment du fédéralisme, effort qu'avaient entrepris les sciences sociales depuis quelques années. On constate pourtant un hiatus entre ses perspectives et celles de la pléiade de jeunes auteurs critiques du tournant de la décennie

21. Nous ne pouvons cependant traiter ici les aspects multiples des prises de position politiques du tournant des années 1980, notre but étant de circonscrire les grandes tendances explicatives. 
1970, moment que nous identifierons comme période waffle $^{22}$. II se manifestait alors chez les spécialistes anglophones des sciences sociales des courants militants qui voulaient prendre le parti des grands mouvements populaires et qui définissaient leur démarche théorique par une volonté de changement politique radical. Ces courants ne furent pas tous associés directement au Waffle néo-démocrate. Mais nous croyons que la référence à une période waffle permet de situer chronologiquement l'impact le plus grand et le moment fort de l'élaboration des paradigmes principaux par lesquels les spécialistes tentèrent de rénover alors l'étude du système politique-économique-social du Canada.

Leur analyse de l'économie politique s'inspirait, à des degrés divers, des théories de la dépendance, et certains voyaient le Canada comme une colonie des États-Unis ${ }^{23}$. Plusieurs ont trouvé dans les organisations revendicatives et le mouvement de la classe ouvrière la puissance de contestation en mesure de porter leurs aspirations. C'est dans ce cadre qu'ils ont abordé la montée, toujours plus évidente à l'époque, du mouvement national au Québec.

\section{L'enthousiasme contestataire}

La première caractéristique des études de cette période est l'absence généralisée de condescendance envers le Québec, typique des anciens points de vue qui, traditionnellement, opposaient l'arriération de la société canadienne-française au caractère dit libéral, moderne, laïque des anglophones du pays. Daniel Drache reprochait ainsi, en

22. Rappelons que le «Waffle» fut une tendance nationaliste de gauche au sein du NPD, que combattit la direction du parti. II apparut publiquement par un manifeste à l'automne de 1969, et ses thèses constituèrent le coeur des principaux débats internes au parti jusqu'à son exclusion du NPD-Ontario, en juin 1972.

23. Une synthèse critique de l'élaboration sur cette question est notamment présente dans : Paul Kellog, "State, Capital and World Economy : Bukharin's Marxism and the "Dependency/Class" Controversy in Canadian Political Economy» in Revue canadienne de science politique, XXII, no 2, juin 1989 , p. 337-362. 
1972, aux «Canadiens anglais» de ne pas voir la part fondamentale qu'avait tenue la classe ouvrière dans l'opposition à Duplessis, et donc de perpétuer une vision tronquée de la vie politique au Québec. «Les orientations de Lesage et Lévesque étaient comprises de manière unidimensionnelle, écrivait-il, par laquelle tout fut interprété en termes de débouchés sociaux et économiques créés pour la classe moyenne.n Cela ne permettait pas de saisir, ajoutait-il, la dynamique beaucoup plus profonde qui secoue le Québec, où la classe ouvrière est amenée à dépasser les perspectives historiques de cette *classe moyennew ${ }^{24}$. Et Drache présentait alors au public anglophone les grands manifestes des centrales syndicales québécoises du début des années 1970.

De fait, et il faut le noter, que ce soit par référence à la "décolonisation", ou par une approche voulant cerner la dynamique "anti-impérialiste» du mouvement indépendantiste, les auteurs progressistes de ce moment cherchèrent à exprimer la dimension de l'autodétermination nationale sousjacente au nationalisme québécois. On ne retrouvera presque plus cette préoccupation au tournant de la décennie 1980. A l'époque, la thèse la plus répandue était celle de "l'antiimpérialisme", que l'évolution idéologique des centrales syndicales québécoises promettait comme courant de masse. Dans ce cadre, retournement complet eu égard aux idées traditionnelles sur le Québec : c'est lui (c'est-à-dire la radicalisation de son mouvement ouvrier) qui devenait un modèle de développement politique et social pour le Canada anglais.

Gary Teeple, dans I'un des livres les plus influents de la période, mettait ainsi en avant que «tout défi réel à la domination du capital, étranger et domestique, doit se fonder comme (...) au Québec sur le segment organisé de la classe ouvrière ${ }^{25}$ ». Dans ce cadre, l'affirmation du droit à

24. Daniel Drache, Québec - Only the Beginning, Toronto, New Press, 1972, p. XVIII-XIX.

25. Gary Teeple, dir., Capitalism and the National Question in Canada, Toronto, University of Toronto Press, 1972, p. XIII. 
l'autodétermination était manifestement à l'ordre du jour, et l'orientation constitutionnelle de P.E. Trudeau pouvait être qualifiée de "fédéralisme des mesures de guerre ${ }^{26}$, cependant que tous les partis fédéraux, y compris les communistes, étaient dénoncés parce qu'ils ne reconnaissaient pas «complètement le droit du Québec à se séparer $^{27}$.

Ce point de vue était articulé à une conception de la société canadienne, qui la définissait d'abord par ses liens de sujétion à la puissance américaine : situation de dépendance économique, politique et culturelle ${ }^{28}$, kévoluant vers un statut colonial dans l'empire américain ${ }^{29}$ ", ou ayant déjà fait du Canada «la plus importante colonie des États-Unis ${ }^{30}$. D'ailleurs, le grand capital indigène s'était lui-même développé en fonction de centres de production essentiellement établis hors des frontières du pays. Pis encore, Michael Bliss allait jusqu'à expliquer que la nature «branch plant" de l'économie canadienne trouvait sa source, paradoxalement, dans les mesures protectionnistes de la National Policy de Macdonald au $\mathrm{XIX}^{*}$ siècle : elles ont simplement conduit les "Américains", soulignait-il, à venir «participer directement à l'économie canadienne ${ }^{31}$. Logiquement, il n'était pas de la nature des élites économiques dominantes du Canada de chercher les modes politiques d'un développement indépendant.

26. Daniel Drache, Québec-Only the Beginning, op. cit., p. XIII.

27. Gary Teeple, op. cit., p. XV.

28. Point de vue qui sert de toile de fond, explicite ou implicite, aux articles que l'on retrouve in : lan Lumsden, dir., Close the 49th Parallel etc. : The Americanization of Canada, Toronto, University of Toronto Press, 1970.

29. Robert Laxer, dir., (Canada) Ltd : The Political Economy of Dependency, Toronto, McClelland and Stewart, 1973, «Forewordx, p. 6.

30. Gary Teeple, op. cit., p. XII.

31. Michael Bliss, "Canadianizing American Business : the Roots of the Branch Plant», in lan Lumsden, dir., op. cit., p. 26-42, citation p. 32. 
A ce sujet, il faut de plus mentionner qu'on ne tend pas alors à conclure, de manière générale, que l'autonomie provinciale représenterait un support privilégié de "l'américanisation". Ce sont les traits caractéristiques de l'économie, des classes possédantes et des institutions politiques qu'elles dominent qui en rendent compte : cela est vrai tout autant pour le gouvernement central que pour les provinces. Bliss, par exemple, prend directement à partie cette thèse voulant qu'un controle par Ottawa des ressources naturelles pourrait en garantir une gestion plus conforme aux visions nationalistes ${ }^{32}$. Des articles de Philip Resnick, John W. Warnock, Frank Peers et Lynn Trainor ${ }^{33}$, notamment, montrent l'intégration du Canada aux États-Unis dans les domaines diplomatique et militaire, et aussi dans la recherche scientifique, la presse parlée et écrite, la télévision, etc. Or, ce sont des domaines qui, en tout ou en grande partie, relèvent de juridictions fédérales.

Cela étant, il y a des nuances à indiquer. L'économiste Cy Gonick écrit que l'analyse de Bliss est juste, mais que I'affaiblissement continu d'Ottawa au bénéfice de Washington a entraîné, de façon concomitante, son affaiblissement face aux provinces ${ }^{34}$. Conclusion partagée par Larratt Higgins, qui, dans Close the 49th Parallel de lan Lumsden, est probablement le spécialiste qui élabore alors le plus directement cette thèse selon laquelle un redressement de la situation passerait par un renforcement des prérogatives d'Ottawa aux dépens des provinces ${ }^{35}$. Mais, même dans cette perspective, ce n'est pas le Québec qui est visé : Cy Gonick soutient que ce sont les provinces anglophones riches

32. Ibid., p. 34.

33. Philip Resnick, «Canadian Defense Policy and the American Empirex, p. 93-115; John W. Warnock, «All the News It Pays to Print», p. 117-134; Frank Peers, "Oh Say, Can You Seew, p. 135-156; Lynn Trainor, «Science in Canada - American Stylew, p. 241-255, in lan Lumsden, op. cit. 73, ibid.

34. C.W. Gonick, «Foreign Ownership and Political Decay», p. 43-

35. Larratt Higgins, «The Alienation of Canadian Resources : the Case of the Columbia River Treaty», p. 223-240, in Ian Lumsden, ibid. 
qui utilisent les demandes légitimes du Québec pour favoriser leur propre intégration continentale, cependant que Higgins ne s'en prend qu'aux orientations du gouvernement créditiste de Colombie-Britannique.

C'était donc, par-delà ces dernières nuances, la nature profonde du système économique canadien qui était incriminée. Robert Laxer en concluait qu'il serait illusoire de se chercher quelque allié «anti-impérialiste» parmi les élites traditionnelles : la lutte pour l'indépendance du Canada est immédiatement une lutte pour le socialisme, sans étape ${ }^{36}$. Et comme les liens de dépendance accroissent dorénavant le poids de tous les problèmes sociaux, tels que le «chômage», la «stagnation industrielle ${ }^{37}$, voire conduisent a la "désindustrialisation" au profit des États-Unis ${ }^{38}$, la classe ouvrière, jugeait-on, allait naturellement être amenée à les remettre en cause.

\section{Le Québec et les étapes}

Pourtant, le refus d'une stratégie étapiste dans le discours nationaliste de gauche n'écartait pas tous les écueils théoriques. Un peu comme si la crainte de la puissance américaine avait semé malgré tout une ambiguïté dans la contestation de l'État canadien. Plus que tout autre, Jim Laxer l'a exprimé clairement.

"Les socialistes d'un pays dépendant, écrivit-il, font face à un problème complexe : ils doivent chercher à arracher le pouvoir des mains des capitalistes domestiques d'une façon qui ne conduit pas seulement à

36. Robert Laxer, «Foreword», op. cit., p. 19.

37. Daniel Drache, *The Canadian Bourgeoisie and Its National Consciousness*, p. 3-25, citations p. 6, in lan Lumsden, op. cit.

38. Robert Laxer, «introduction», p. 20; Jim Laxer, «Canadian Manufacturing and U.S. Trade Policy», p. 127-147; Jim Laxer et Doris Jantzi, *The De-Industrialization of Ontario», p. 147-152, in Robert Laxer, op. cit. 
renforcer le pouvoir des compagnies étrangères aux dépens des intermédiaires locaux. (...) (Les) socialistes canadiens doivent se doter d'une orientation qui évite deux dangers : renforcer le capitalisme d'État, par une peur trop grande pour le sort des institutions canadiennes; et faciliter la mainmise américaine, en visant le pouvoir d'une manière qui ne conduit qu'à affaiblir ces institutions et à miner la confiance du peuple canadien en sa capacité de résistance ${ }^{39}$.ر

Théoriquement, il n'y avait pas d'étape (nationale) dans la lutte pour le socialisme, mais concrètement, il y a eu tendance à privilégier l'opposition aux États-Unis. Mel Watkins écrivit ainsi que ul'ennemi ultime est l'impérialisme U.S. et non le capitalisme anglo-canadien dépendant ${ }^{40}$; ; et lorsqu'il critique la position du syndicalisme canadien sur la question nationale en 1973, ce n'est pas de son orientation sur le Québec qu'il traite, mais de la faiblesse de ses engagements envers la souveraineté de l'État canadien ${ }^{41}$.

Dès lors, c'est sans doute par besoin tant stratégique que théorique que Jim Laxer rédigea cette même année un article important sur le Québec ${ }^{42}$. II y explique les revendications québécoises par les conditions historiques et politiques d'inégalité faites aux francophones; refusant de

39. Jim Laxer, $*$ The Americanization of the Canadian Student Movementw, p. 275-286, citation p. 278, in lan Lumsden, op. cit.

40. Mel Watkins, "Contradictions and Alternatives in Canada's Futurew, p. 250-269, citation p. 262-263, in Robert Laxer, op. cit.

41. Mel Watkins, *The Trade Union Movement in Canada», p. 178-196, voir les pages 187 sqq, in Robert Laxer, ibid.

42. Jim Laxer, «Québec in the Canadian Federal State», p. 232 249, in Robert Laxer, ibid. 
considérer le Canada français comme un bloc homogène, il rappelle que les mouvements populaires libéraux $y$ ont été battus par des forces cléricales «soumises à leurs maîtres anglais». Dénonçant les campagnes que menait George Brown au temps de l'Acte d'Union pour I'application exclusive du principe de la «représentation selon la population» (rep. by pop.), Laxer souligne qu'elles étaient kune version précoce de la politique d'un Canada unique, pour laquelle la démocratie (dans ce pays) est simplement une question de droits individuels et $n$ 'implique pas les droits des collectivités nationales qui forment le Canada». Au sujet du nationalisme québécois contemporain, Jim Laxer définissait l'idéologie du PO comme relevant des orientations d'une nouvelle classe moyenne, qui voulait obtenir directement pour sa province (sans passer par l'intermédiaire d'Ottawa) le statut qui est celui du Canada "dans l'empire américain". Mais il ne restreignait pas à cette idéologie le sens des revendications nationales. Étudiant la montée des mobilisations ouvrières au Québec durant les années 1960 et au tournant de la décennie suivante, il se montrait particulièrement enthousiasmé par les réalisations des fronts communs syndicaux. II en déduisait, lui aussi, qu'une "lutte significative ouvrière et antiimpérialiste pour un Québec indépendant est née».

La conclusion stratégique que tirait alors Laxer apparaît extrêmement symptomatique de l'ambiguïté que nous avons relevée dans la disposition politique du nationalisme canadienanglais de gauche face à l'État. Laxer pensait qu'il était dorénavant réaliste d'espérer "que les Canadiens anglais et les Québécois ne soient plus les victimes d'un système fédéral qui a simplement livré les deux parties du pays aux compagnies américaines» et qu'une opposition "simultanée» à la mainmise des États-Unis sur les ressources naturelles «de la moitié septentrionale de ce continent" pourrait donner I'occasion "d'une alliance anti-impérialiste ${ }^{43}$ ".

En d'autres termes, à la fin d'une analyse où il avait soutenu que la question nationale du Québec était comprise dans I'histoire même de l'État au Canada, Jim Laxer proposait

43. Les citations de Laxer sont tirées de : ibid, p. 233, 244-45, 
une lutte conjointe contre la puissance américaine, et non contre cet État canadien. Théoriquement, il n'y avait pas "d'étapes" dans l'analyse de la gauche anglophone mais, en pratique, c'était l'emprise des États-Unis qui était visée.

Nous ne voulons pas démontrer par là que les intellectuels de la période waffle étaient retournés malgré tout à une position chauvine sur le Québec : ce serait faux. Quand Mel Watkins, par exemple, cite deux menaces cruciales à la «survie du Canadan, il mentionne l'empire américain et la "crise des relations" entre le Québec et le Canada anglais, qu'il fait reposer sur l'incapacité des anglophones à accepter les réalités nationales véritables du pays - et non sur le nationalisme québécois, la nuance est importante ${ }^{44}$. Mais il se dégage néanmoins des analyses d'alors la constante d'une hésitation politique dans la contestation de l'État canadien, qui rendait à terme improbable l'articulation d'une alliance profonde avec le nationalisme québécois.

Cela étant dit, il semble que les points de vue élaborés a la fin de la décennie 1970 , et que nous avons illustrés précédemment en centrant l'attention sur les contributions de Whitaker et Stevenson, ont comporté à la fois des éléments de continuité mais aussi de rupture avec les positions des auteurs de la période waffle. Par exemple, il est évident que le nationalisme québécois est vu beaucoup moins positivement et même avec méfiance chez certains; d'un autre côté, la prééminence des préoccupations pour l'avenir de l'État canadien s'affiche explicitement, de façon moins critique qu'au début de la décennie. Plusieurs facteurs rendent compte de ces éléments de rupture et de continuité analytiques, dont nous voudrions indiquer brièvement quelques aspects.

Gerald L. Caplan et Jim Laxer ont expliqué déjà que la «revitalisation" de la pensée socialiste au Canada anglais dans les années 1960 était liée à la "question nationale» (c'est-àdire le refus de l'emprise américaine), et que celle-ci avait

44. Mel Watkins, «Contradictions and Alternatives in Canada's Future», op. cit., p. 262-263. 
constitué ala première de ses priorités ${ }^{45}$. Idéologiquement, le nationalisme est manifestement devenu, sur cette base, I'un des traits les plus répandus de l'analyse critique des sciences sociales au Canada anglais. Par ailleurs, les grands syndicats québécois $n$ 'ont finalement pas cherché à assumer par eux-mêmes, en un projet et une organisation politiques indépendants, les aspirations nationales du Québec. Les proclamations radicales et kanti-impérialistes» de leurs manifestes, qui avaient tant enthousiasmé la génération waffle, sont passées au second plan. Pour plusieurs des auteurs progressistes de la fin de la décennie 1970, il semble que ces deux éléments se soient alors conjugués à l'ambiguïté théorico-stratégique de l'analyse waffle sur l'État canadien, produisant des points de vue pour lesquels la sauvegarde de cet État apparaît comme l'une des premières préoccupations, qui conditionne même la perception des autres réalités. On est moins bien disposé face au Québec, et on tend à ramener le sens des développements nationaux de sa population à l'origine sociale de la direction du $\mathrm{PQ}$ ou à la nature de son programme. II y a donc des éléments de rupture dans l'appréciation des revendications autonomistes du Québec, mais ils sont inséparables de la perpétuation, voire de l'essor, du sentiment de lien à l'État canadien, dont la période waffle avait fait montre et qu'elle avait étoffé.

\section{L'opposition à l'Accord du lac Meech}

Dans la première moitié des années 1980 , la principale particularité des analyses effectuées par l'intelligentsia anglophone progressiste sur les problèmes de constitution semble être leur nombre très réduit. Ce sera au contraire à une pléthore d'interventions, de livres et d'articles que donnera naissance l'entente du lac Meech, par lesquels, en règle générale, cette intelligentsia a manifesté formellement son rejet de ce nouveau projet de modification de l'AANB. Mais alors qu'au début de la décennie 1970 on aurait pu

45. *The first item on its agendax, écrivent-ils. Gerald L. Caplan et James R. Laxer, «Perspectives on Un-American Traditions in Canada», in lan Lumsden, op. cit., p. 305-320;voir la page 310. 
espérer la formation d'un terrain d'élaboration constitutionnelle commun aux sciences sociales critiques du Canada anglais et du Québec, vingt ans plus tard, le cycle des crises contemporaines de la Confédération semblait devoir se clore sur une antinomie extrême des points de vue.

\section{A la recherche du Québec}

Dans son introduction au recueil de textes sur la réforme constitutionnelle de 1981-1982 qu'il a publié pour la Société d'études socialistes de Winnipeg ${ }^{46}$, Robert Martin soulignait que les contributions colligées visaient à évaluer la constitution nouvelle et sa Charte des droits et libertés du point de vue du progrès social et des impératifs de la reconnaissance des droits des femmes, des autochtones et du peuple québécois. Son propre article, centré sur les garanties accordées à l'exercice des droits et libertés au Canada, mettait l'accent sur la nette insuffisance des dispositions de la Charte. Ces droits et libertés sont «présentés sous forme d'abstractions vagues", écrivait-il, ce qui forcément limite leur signification; ils doivent de plus compter avec la section première de la Charte, qui les soumet à la possibilité de «restrictions illimitées» (open-ended qualifications). A cela $s$ 'ajoute l'arbitraire politique qui préside à la nomination des juges au Canada. Martin voyait difficilement, en conséquence, comment les cours de justice allaient - plus qu'auparavant - être en mesure d'agir à titre de défenseurs des droits contre les gouvernements ${ }^{47}$.

Leo Panitch et Donald Swartz adressaient à la Charte une critique similaire : prétendre, en cette fin de $X X^{\bullet}$ siècle, reconnaître le droit d'association sans mentionner explicitement le droit de négocier et de faire grève, $n^{\prime}$ offre au mouvement ouvrier aucune garantie véritable. Le droit d'association est ainsi défini si kabstraitement", qu'il est

46. Robert Martin, dir., Critical Perspectives on the Constitution. Socialist Studies/Études socialistes, no 2, Society for Socialist Studies Société d'études socialistes, Winnipeg, 1984.

47. Robert Martin, «The Judges and the Charter», p. 66-83, ibid. 
dépouillé des seuls attributs qui lui confèrent aujourd'hui sa substance sociale $^{48}$. Même position du côté des critiques féministes : Gayle Raphanel soulignait en 1984 que la Charte de la nouvelle loi constitutionnelle ne permettait pas d'atteindre l'égalité de fait avec les hommes parce qu'elle ne fondait pas l'adoption de mesures de redressement positives de la situation des femmes ${ }^{49}$. Chaviva Hosek, alors dirigeante féministe et par la suite ministre de l'Habitation dans le gouvernement Peterson en Ontario, notait que lors des débats sur l'enchâssement d'une charte des droits, des groupements de femmes avaient manifesté leur appui à l'adoption d'un tel document parce qu' on voulait se protéger de certaines provinces particulièrement rétrogrades. D'autres craignaient pourtant l'intervention des juges, dont le conservatisme traditionnel au Canada était bien connu, et préféraient se fier aux avancées de nature politique. Mais le premier projet de charte leur apparut tellement régressif, que toutes jugèrent finalement nécessaire d'intervenir pour le corriger. Chaviva Hosek considérait que le résultat était partagé, mais elle se disait «troublée» par l'envergure des efforts qui avaient été nécessaires pour que soit simplement garantie l'égalité des droits ${ }^{50}$.

II y avait donc des éléments de critique de la charte fédérale sur le terrain même des droits qu'elle est censée garantir. Le processus du renouvellement constitutionnel (réservé aux instances de l'État, sans participation décisionnelle de la population) fut à l'occasion également dénoncé mais non massivement, et pas du tout sous la forme d'une implication suivie, dans les années immédiatement postérieures à 1982, sauf du côté des autochtones.

48. Leo Panitch et Donald Swartz, The Assault on Trade Unions, Toronto, Garamond Press, 1988, notamment les pages 48, 55 et 65 .

49. Gayle Raphanel, «Justice and Sexual Politics*, p. 125-130, in Robert Martin, op. cit.

50. Chaviva Hosek, "Women and the Constitutional Process", in K. Banting et R. Simeon, dir., And No One Cheered, Toronto, Methuen, 1983, p. $280-300$. 
Dans un court article rédigé pour le recueil de Robert Martin, Reg Whitaker souligne ce manque d'intérêt évident de la gauche anglophone pour les questions soulevées par le rapatriement de la constitution. Quant à lui, écrivait-il, il entendait cette fois traiter des conséquences précises de la réforme de 1982 sur les krapports constitutionnels entre le Canada français et le Canada anglais». Et il arrivait à la conclusion que la nouvelle loi constitutionnelle et sa charte représentaient «un compromis raisonnable entre des pressions d'origines multiples", principalement entre la volonté du Québec de maintenir sa Loi 101 et l'application universelle des clauses de la charte fédérale : le gouvernement Trudeau avait pris garde, en fin de course, qu'elle ne puisse empêcher Québec de légiférer pour que l'environnement social et économique de la province soit francisé ${ }^{51}$. Cette évaluation posée, Whitaker expliquait le xconservatismen de la constitution réformée par le fait, notamment, que «la gauche canadienne elle-mêmen s'était avérée «incapable de profiter de l'ouverture (qui s'est présentée en 1981-1982) pour mettre en avant" des revendications démocratiques plus exigeantes $^{52}$.

C'était là un élément important, illustration d'un véritable tournant sur le point de s'engager dans les considérations constitutionnelles dominantes de l'intelligentsia anglophone progressiste. Le professeur Alan C. Cairns, de la Colombie-Britannique, a été l'un des premiers analystes à se pencher sur l'utilisation par le gouvernement Trudeau d'une politique d'enchâssement des droits et libertés afin d'affermir la position d'Ottawa face aux provinces. Plus précisément, il a montré que le gouvernement central s'est servi au début des années 1980 du projet d'une charte des droits afin de se créer une base politique au sein de la population (constituency) : n'eût été de ce projet, son «unilatéralisme se serait réduit à un

51. Reg Whitaker, «Democracy, Federalism and the National Political Communities in Canada», in Robert Martin, op.cit., p. 255-268.

52. Ibid., p. 267. 
simple jeu de pouvoirn, sans appui populaire ${ }^{53}$. Ce constat ne supposait chez Cairns aucune évaluation négative. Dans la seconde moitié de la décennie, il soutiendra plutôt que l'un des mérites de la Charte fut précisément de faire en sorte que les questions de constitution cessent de n'intéresser que les spécialistes et les gouvernements, et aussi de ne porter que sur des rapports de juridiction. A partir de 1981-1982, on comprit aussi que les débats constitutionnels impliquaient des choix de société et des principes : "égalité des sexes", droits des autochtones, minorités visibles, etc. ${ }^{54}$. Le tournant dont nous avons parlé, c'est que le Québec en vint progressivement, sur cette base, à disparaître presque des préoccupations des analystes de la gauche anglophone. Pas encore toutefois chez Reg Whitaker, mais très certainement dans les points de vue qui vont devenir hégémoniques à la fin de la décennie 1980.

Déjà, l'appréciation globale que portait Cairns sur l'issue même des conflits constitutionnels de 1981-1982 était marquée par le désintérêt à l'égard de la dimension québécoise. Deux éléments centraux ressortaient en effet. D'abord, le "système constitutionnel canadien» avait fait preuve, disait Cairns, d'un «conservatisme» prononcé, d'une grande "stabilité", malgré les volontés multiples de le modifier. Ensuite, la conclusion concrète des débats en 1982 representait un compromis entre les deux grandes tendances qui s'étaient affrontées, les provinces acceptant la Charte (dont elles réussirent cependant à "affaiblir» l'effet "nationalisateurn) et Ottawa faisant des concessions sur la

53. Alain C. Cairns, «The Politics of Constitutional Conservatism», p. 28-58, notamment les pages $39-42$ et 53 au sujet de la Charte, in K. Banting et R. Simeon, op. cit.

54. Voir à ce sujet la référence que fait a Cairns le professeur Thomas J. Courchesnes, «Meech Lake and Socio-Economic Policy», p. 63-81 in L'Accord du lac Meech. Canadian Public Policy/Analyse de politiques, numéro spécial XIV, Supplément, préparé sous la direction de K. Norrie et F. Vaillancourt, Guelph, sept. 1988, notamment p. 64 . 
formule d'amendement constitutionnel (bien que pas autant que les provinces $n$ 'auraient souhaité) ${ }^{55}$.

Notons bien : Cairns caractérisait la loi de 1982 en ne s'arrêtant qu'à l'entente entre les provinces anglophones et Ottawa, même si on avait laissé de côté le Québec dont les aspirations étaient à l'origine du malaise constitutionnel et du processus de révision de l'AANB. II voyait sous l'angle unique du *conservatisme» la «stabilité» du système constitutionnel malgré que l'orientation d'Ottawa ait eu préséance sur la volonté unanime de l'Assemblée nationale.

Reg Whitaker avait parlé de compromis en 1981-1982 parce que Québec pouvait notamment maintenir ses politiques linguistiques - en d'autres mots parce que la réforme constitutionnelle, pensait-il, assurait à la province certains acquis autonomistes. II déplorait, par ailleurs, que les progressistes n'aient pas participé davantage à l'élaboration de la Charte des droits. Cairns, de son côté, pensait aussi que la réforme d'alors était un «compromis", mais à partir d'une appréciation uniquement centrée sur les résultats du choc de visions concurrentes entre des provinces et Ottawa, sans tenir compte des questions nationales. II semble que pendant un court moment, grosso modo entre 1983 et 1987, il n'y ait pas eu vraiment de point de vue dominant au sein de la gauche universitaire anglophone sur ces questions. $\dot{A}$ l'occasion des débats sur l'Accord du lac Meech, il devint cependant évident que la recherche de formules pouvant répondre positivement aux demandes du Québec n'était théoriquement plus considérée par la majorité de cette gauche comme l'un des enjeux premiers des réformes constitutionnelles. Le tournant qui s'annonçait avec certaines études de la loi de 1981-1982 était dorénavant réalisé, en définissant pour la génération critique actuelle un paradigme global généralement conforme à l'approche de Cairns.

55. Alan C. Cairns, «The Politics of Constitutional Conservatism*, op. cit., p. 28-43. 
Fódéralisme et dualité

A notre avis, ce sont d'ailleurs les articles récents du professeur Alan C. Cairns qui condensent et articulent de la manière la plus expressive les tenants et aboutissants de ce paradigme, même si habituellement le professeur Cairns n'est pas identifié aux courants de gauche des sciences sociales. Nous nous servirons donc de ses principaux écrits sur le sujet.

La thèse centrale de Cairns part de cette notion qu'il y aurait dorénavant opposition au Canada entre deux grandes visions des questions de constitution, kqui toutes deux s'inspirent de la loi constitutionnelle de 1982" (souvenonsnous que, pour Cairns, cette loi fut un «compromisn). Une première vision, selon laquelle ces questions relèvent fondamentalement des gouvernements et visent d'abord en régime fédéral à régler leurs conflits de juridiction; cette vision est notamment portée par la formule d'amendement adoptée en 1982. Et une deuxième vision qui découle plutôt des principes de la Charte des droits et libertés pour laquelle la constitution est l'affaire des citoyens qui veulent dorénavant agir à titre de "participants" du «changement constitutionnel» plutôt que d'en être les "observateurs" ${ }^{50}$. Le problème que pose I'Accord du lac Meech, c'est qu'il avait tout entier été négocié, dit Cairns, selon la seule première vision, sans aucun input populaire, phénomène cristallisé dans la formule d'amendement mise au point, qui ne se référait qu'à l'exigence de l'unanimité des gouvernements. L'Accord du lac Meech, concluait-il, était en ce sens «illégitime du point de vue d'une constitution des citoyens".

Les articles de Cairns démontrent bien le caractère non démocratique des processus de négociation ayant conduit à la rédaction de l'Accord et, pour lui, I'hostilité que l'entente a suscitée chez les groupes populaires et ouvriers vient de ce

56. Alan C. Cairns, "Citizens (Outsiders) and Governments (Insiders) in Constitution-Making: The Case of Meech Laken, p. 121-145, citation p. 121 in L'Accord du lac Meech. Canadian Public Policy/Analyse de politiques, op. cit. Voir aussi, exprimant le même point de vue, l'article de Cairns intitule *The Limited Constitutional Vision of Meech Lakew, in K.E. Swinton et C.J. Rogerson, dir., Competing Constitutional Visions : The Meech Lake Accord, Agincourt, The Carswell Co. Ltd, 1988, p. 247-262. 
que ceux-ci, depuis 1982, se sentent directement concernés par les débats constitutionnels. Ce dernier élément est fondamental. Selon I'explication de Cairns, c'est la Charte qui a éveillé dans la population canadienne ce que nous pourrions appeler le sens de la citoyenneté démocratique et la vigilance constitutionnelle. C'est ainsi qu'il parle de Charter Canadians (c'est-à-dire les citoyens nés de et avec la Charte), et qu'il écrit : «avec cette culture constitutionnelle de 1982, et en particulier de la Charte, le controle gouvernemental sur les processus d'amendement, qui autrefois était considéré comme allant de soi, est maintenant vu comme un élitisme $\operatorname{arrogant}^{57}$.

Dans ce cadre, les demandes autonomistes du Québec s'inscrivent obligatoirement dans les préoccupations de la première vision, celles qui ne relèvent pas de la démocratie. Ce n'est pas là une interprétation de notre part. L'auteur écrit, par exemple, que ula Charte(...) passait outre les gouvernements et s'adressait directement aux Canadiens en les définissant comme titulaires de droits, aussi bien qu'en accordant une reconnaissance constitutionnelle spécifique ${ }^{58}$ " à certains groupes particuliers : femmes, minorités linguistiques, etc. La Charte ne reconnaît pas, on le sait, le droit du Québec à l'autodétermination, mais Cairns ne le mentionne nulle part : manifestement, il ne voit pas là un accroc à la démocratie et il réduit donc la question du statut du Québec à la mécanique des rapports entre instances de la fédération. À ce sujet, il est nécessaire aussi de mentionner, même brièvement, les deux éléments suivants.

Aucun des effets positifs que Cairns attribue à la Loi constitutionnelle de 1982 n'a été interprété comme tel au Québec par les groupes progressistes. La Charte fédérale n'a jamais été considérée par eux sous l'angle d'une inspiration; elle a été plutôt envisagée comme partie prenante d'un «coup

57. Alan C. Cairns, «Ottawa, the Provinces and Meech Lakew, in Roger Gibbins, dir., Meech Lake and Canada : Perspectives from the West, Edmonton, Academic Printing and Publishing, 1988, p. 105-117, citation p. 117.

58. Alan C. Cairns, «Citizens (Outsiders) and Governments...»,op. cit., p. 122. 
de force" constitutionnel, ressenti comme la négation des principes de la démocratie. De même, lorsque ces groupes se sont opposés à l'Accord du lac Meech, ce n'est pas en se fondant sur la Charte de 1982, mais parce que I'Accord perpétuait une histoire constitutionnelle décriée - dont la Charte fait partie ${ }^{59}$. Par ailleurs, nous croyons que cette analyse elle-même de la Charte est discutable, y compris pour le Canada anglais. II faut rappeler que la Charte de 1982 (comme le souligne à sa façon son article premier) est un document octroyé par les pouvoirs publics : ultimement, elle ne signifie pas la préminence des droits des citoyens sur les gouvernements, mais une extension de la «franchise» consentie par ces gouvernements à leurs sujets, illustrée par le fait que c'est en audiences, sous forme de consultation, que la population a pu se faire entendre à son sujet. Les juristes débattent encore de la portée exacte des droits qu'elle consigne, mais il est difficile de saisir en quoi elle reposerait sur une avancée radicale des principes de la souveraineté populaire, seule signification démocratique véritable qu'on pourrait accoler à l'expression «constitution des citoyens".

Quoi qu'il en soit, la Charte et la Constitution de 1982 étaient maintenant parées de toutes les vertus comparativement à l'Accord du lac Meech. Non seulement ne se préoccupait-on plus des termes de la coexistence constitutionnelle des groupes nationaux, mais encore écrivaiton que la mention explicite de «l'existence de Canadiens d'expression française et d'expression anglaise", ou la «reconnaissance que le Québec constitue une société distincte à I'intérieur du Canada", dans I'Accord du lac Meech, paraissaient contredire la notion $* d$ 'un statut constitutionnel égal pour toutes les composantes de la société canadienne», établie par la Charte de $1982^{60}$. En outre, le caractère

59. Voir la position des centrales syndicales du Québec et, en particulier, les extraits significatifs du texte par lequel la FTO s'est opposée officiellement à l'Accord du lac Meech, rapportés in L'Accord du lac Meech, textes réunis par une équipe du journal Le Devoir, Montréal, Guérin, 1987. p. 233-237.

60. Alan C. Cairns, «Citizens (Outsiders) and Governments...», op. cit., p. 130. 
centralisateur de la réforme constitutionnelle promulguée cette année-là devenait une donnée structurante des garanties démocratiques, puisque la Charte repose sur le "pancanadianisme» et en procède ${ }^{\circ 1}$. L'on ne retrouvait qu'à peine ces quelques critiques adressées à la Charte fédérale pendant la première moitié de la décennie 1980, notamment en ce qui concerne les droits des femmes.

Cette évolution en est venue à produire (reproduire?) des réflexes qui présentent même des traits désobligeants. Par exemple, dans une étude qui n'est pourtant pas un pamphlet, A. Wayne MacKay ${ }^{62}$ a voulu mesurer l'impact qu'aurait eu sur l'interprétation de la constitution la reconnaissance de la "société distincte" et de la «dualité linguistique» dans I'Accord du lac Meech. Selon lui, cet impact aurait été réel, "ces additions" à la constitution ne représentant pas «qu'un énoncé de nature sociologique». Soit, mais ce qui est alors vraiment surprenant, c'est que I'auteur concluait, sans autre forme de démonstration, qu'elles auraient donc «limit(é) les droits inscrits dans la Charte, bien qu'il ne soit pas encore évident de quelle manière et jusqu'à quel point»! Pourquoi la reconnaissance de la "société distincten, si elle avait eu une portée réelle, n'aurait-elle pas conduit plutôt vers un élargissement du champ des libertés? Cette hypothèse n'a pas été envisagée, mais une conclusion contraire a été tirée. De même, Kathleen Mahoney a pour ainsi dire écrit que toute reconnaissance de droits nationaux au Québec pourrait entraîner la négation des droits individuels et collectifs partout au Canada, particulièrement des droits des femmes ${ }^{63}$. Sur la base, par exemple, de l'accès à l'avortement depuis vingt ans ou de l'orientation référendaire des "Yvettes", on se demande pourquoi, à tout le moins et

61. Ibid., p. 135.

62. A. Wayne MacKay, *Linguistic Duality and the Distinct Society in Québec : Declaration of Sociological Fact or Legal Limits on Constitutional Interpretation», p. 65-79, in K.E. Swinton et C.J. Rogerson, op. cit., voir en particulier la page 78 pour les citations qui suivent.

63. Kathleen Mahoney, «Women's Rights*, in Roger Gibbins, op. cit., p. 159-170. 
encore une fois, une plus grande autonomie québécoise n'était pas vue aussi comme pouvant aller de pair avec d'importantes conquêtes démocratiques, par exemple le fait d'exempter le Québec de majorités influencées par les Vander Zalm, le Reform Party, la législature du Manitoba, etc. sur la question même du libre choix, ou encore, a l'époque, des projets législatifs du gouvernement Mulroney visant à recriminaliser l'avortement.

En ce qui a trait à la discussion sur l'Accord du lac Meech, ces quelques éléments permettent de cerner la position probablement majoritaire des universitaires progressistes de langue anglaise, qui les a conduits tout droit à un point de vue achevant un cycle : non seulement ils ne tenaient plus compte des revendications a I'autodétermination, non seulement ils identifiaient la promotion des droits au renforcement de l'État central, mais encore tendaient-ils à définir les conditions mêmes de l'exercice de la démocratie et de la défense des acquis sociaux sur un axe excluant les aspirations nationales. Cela, c'était nouveau comme paradigme (nous semble-t-il) hégémonique.

Jim Laxer, on l'a rappelé plus haut, avait réprouvé au début des années 1970 cette position d'un "Canada unique", selon laquelle "la démocratie est simplement une question de droits individuels et $n^{\prime}$ implique pas les droits des collectivités nationales qui forment le Canadan. Nous pensons que la gauche universitaire du Canada anglais était alors revenue à cette case départ, dénoncée à juste titre par Laxer il y a quelque vingt ans : elle définissait majoritairement la démocratie, ses acquis et ses revendications selon une trajectoire qui ne peut faire droit aux demandes de la "collectivité nationale" québécoise. Alors que les centrales syndicales du Québec, notamment, refusaient l'Accord du lac Meech parce qu'il ne (se) fondait pas (sur) l'exercice effectif de l'autodétermination, les progressistes anglophones réclamaient, en pratique, une négation plus crue de ses principes. 


\section{Une conclusion en forme de prémisse}

L'attachement à l'État canadien a été une constante de la pensée progressiste au Canada anglais durant le dernier quart de siècle. Politiquement, il remonte d'ailleurs loin dans I'histoire de la gauche et trouve des fondements au sein de l'évolution du dominion canadien, comme les travaux de Norman Penner l'ont montré ${ }^{04}$. Mais cet attachement s'est affiché encore plus ouvertement avec les années 1980. L'adjonction de la Charte des droits et libertés au processus de rapatriement de l'AANB a réussi à associer, dans l'esprit d'une majorité des intellectuels progressistes, les succès revendicatifs à la centralisation de la Confédération et à la préséance d'Ottawa sur les provinces ${ }^{05}$. La signature du Traité de libre-échange avec les États-Unis a par ailleurs exacerbé la peur de l'absorption dans l'empire américain et le sentiment d'insécurité culturelle, poussant encore davantage au désir d'un renforcement des institutions établies. De sorte que la pensée progressiste a eu tendance à se lier aux formes de l'État constitué, et à s'y limiter.

64. Par exemple, le type d'intervention (et les difficultés idéologiques) qu'entraîne pour le Parti communiste la question de I'indépendance du Canada. Voir de Norman Penner, The Canadian Left : A Critical Analysis, Toronto, Prentice-Hall, 1977.

65. C'est le sens même que prend l'article récent de Katherine Teghtsoonian, Neo-Conservative Ideology and Opposition to Federal Regulation of Child Care Services in the United States and Canadaw, in Revue canadienne de science politique, XXVI, no 1, mars 1993, p. 97-121, où l'auteure conclut à un lien étroit entre la promotion des valeurs néoconservatrices et celle du respect des juridictions provinciales, ne s'arrêtant pas à la position des groupes de femmes du Québec qui ont plutót expliqué, précisément, que le réseau de garderies au Québec était mieux défendu par la garantie d'une juridiction exclusive de la province, parce qu'Ottawa avait une orientation d'aide aux utilisateurs/trices par la méthode des crédits d'impót alors que Québec intervenait par celle de l'aide a la création et au financement des établissements, que favorisent partout les organisations féministes (voir la présentation de la Fédération des femmes du Québec au Comité mixte du Sénat et de la Chambre des Communes sur l'Accord du lac Meech, reproduit in Barbara Roberts, Beau fixe ou nuages l'horizon?, Feminist Perspectives féministes, 12b, février 1989, ICREF, Ottawa, p. 2327. 
Or, l'effet de la réforme constitutionnelle de 1982 a été diamétralement contraire au Québec, l'opération du «rapatriement unilatéral» $y$ ayant accentué le sentiment d'aliénation des groupes syndicaux, de gauche, etc., envers l'État fédéral. Les courants progressistes des deux groupes linguistiques s'engageaient ainsi dans des démarches qui ne pouvaient trouver de correspondances. Pourtant, le fait que les libéraux et les péquistes, ensemble a l'Assemblée nationale, aient appuyé le libre-échange avec les États-Unis a donné l'impression qu'il y avait unanimité au Québec en faveur de cette politique du gouvernement Mulroney. Tous éléments dont la conjonction a articulé une atmosphère d'irascibilité envers le Québec, la première pièce marquante ayant été l'ouvrage de Philip Resnick, Letters to a Québécois Friend $^{\circ 6}$. Et quand on relit ces lettres avec quelques années de recul, ce qui saute aux yeux, c'est bien le reproche adressé aux Québécois de ne pas montrer les mêmes dispositions que les anglophones face aux institutions canadiennes ${ }^{67}$.

Cette fois, tous les coups semblèrent permis à certains, y compris les menaces contre l'intégrité territoriale du Québec s'il s'aventurait à exercer son droit à l'autodétermination. Politiquement, la pointe avancée de cette logique fut acérée par Mel Hurtig, chef du Parti national du Canada, qui affirme que «les députés du Bloc québécois qui ne prêtent pas serment d'allégeance au Canada ne devraient pas avoir le droit de siéger au Parlement. "Je ne veux pas que l'argent des contribuables serve à soutenir la cause de ces gens qui veulent détruire notre pays ${ }^{n 8}$ ". Pour Mel Hurtig, membre

66. Philip Resnick, Letters to a Québécois Friend. With a Reply by Daniel Latouche, Montréal et Kingston, McGill-Queen's University Press, 1990. Il existe une version française publiée la même année par les éditions du Boréal de Montréal.

67. Cf. notre lecture critique de ces lettres et de la réponse de Daniel Latouche in Recherches sociographiques, XXXIII, no 2, 1992, p. 337 342.

68. La Presse, 08/05/93, p. D20. Notons que la dépêche soulignait : $*$ M. Hurtig a dit que, partout au pays, plus que toutes les autres politiques de son parti, ce sont ses prises de position contre les séparatistes qui réjouissent les Canadiens»! 
fondateur du Conseil des Canadiens, la légitimité des parlementaires ne semble donc pas procéder de leurs commettants, mais de la soumission aux institutions établies. Caricature du nationalisme anglo-canadien dit progressiste, extrémisme? Sûrement, mais position qui n'est pas qualitativement différente de celles qui annoncent des prétentions sur le territoire québécois. L'émotivité qui porte ces points de vue est un danger évident pour l'avenir même de la démocratie au Canada anglais : on n'ose imaginer la réaction d'une certaine gauche s'il y survenait une «affaire Boudare $\left.\right|^{69}$.

Heureusement, pourrions-nous dire, l'agitation constitutionnelle a tout de même permis (ou forcé) de nouvelles percées idéologiques et théoriques. L'état de crise récurrent du fédéralisme canadien soumet les schèmes conceptuels par lesquels sont abordés les problèmes de constitution à des questionnements répétés, apportant obligatoirement des éléments d'instabilité. En ce sens, il n'est pas accidentel que ce soit du côté d'un grand mouvement social - confronté à la nécessité continuelle de se déclarer politiquement - que soit apparu, au cours des deux dernières années, le renouveau le plus spectaculaire. À l'été 1991, le congrès du Comité canadien d'action sur le statut de la femme (CCA; National Action Committee on the Status of Women - NAC) adoptait en effet une résolution constitutionnelle qui tranchait avec les points de vue élaborés par la majorité des progressistes anglophones durant la

69. Georges Boudarel est un universitaire français qui, jeune agrégé de philosophie, fut envoyé en 1947 pour son premier poste d'enseignement en Indochine. Révolté par l'intervention des troupes coloniales de la France, il rejoignit le Viet Minh et devint même instructeur politique dans un camp de prisonniers français. Au début de l'année 1991, éclata en France ce qu'il est convenu d'appeler «l'affaire Boudarel» quand, avec deux livres autobiographiques (Autobiographie, Paris, Jacques Bertoin éditeur, 1991; Cent fleurs écloses dans la nuit du Vietnam, Paris, Jacques Bertoin éditeur, 1991), celui-ci rappela publiquement qu'il avait alors pris parti pour le droit des peuples contre son pays. Le professeur Jean Chesneaux, qui a analysé «l' affaire» pour Le Monde diplomatique (numéro de mai 1991, p. 32) souligne comment «l'extrême-droite» s'est alors «déchaînée» contre Boudarel, dans un climat «néomaurassien*, et comment l'universitaire fut soumis à un véritable «lynchage médiatique». 
seconde moitié de la décennie 1980. Non seulement y a-t-on reconnu le Québec comme «nation*, mais on a adopté des résolutions statuant que le peuple québécois et les aborigènes devaient décider par eux-mêmes et xunilatéralement" de leur sort, leurs décisions devenant par la suite la base acceptée de négociations avec le Canada anglais ${ }^{70}$.

Évidemment, on ne peut prévoir à long terme l'impact de cette prise de position, mais il faut tout de même noter qu'elle a permis, à l'automne 1992, au CCA-NAC de se définir en toute autonomie politique face a I'Accord de Charlottetown. Le Comité fit activement campagne en faveur du non référendaire, contrairement au Congrès du travail du Canada (CTC), par exemple, qui, parce qu'il croyait cela nécessaire à la sauvegarde du pays, se rangea à nouveau du côté d'un projet constitutionnel mis au point par les partis traditionnels. Ce faisant, soit dit en passant, le CCA-NAC adoptait une position compatible avec celle des organismes populaires et syndicaux du Québec, une nouveauté dans les rapports entre groupes progressistes des deux collectivités linguistiques depuis près d'un quart de siècle.

Ce sont sûrement les mêmes circonstances qui ont amené Philip Resnick en 1991, dans son essai Toward a Canada-Quebec Union ${ }^{71}$, et Daniel Drache et Roberto Perin en 1992, dans Negotiating with a Sovereign Quebec ${ }^{72}$, a revoir et réévaluer certains des fondements les plus répandus des analyses progressistes publiées au Canada anglais sur la question de l'État fédéral et du Québec. Explicitement, ces auteurs ont centré leur effort d'analyse sur un point de vue qui ne stipulait plus, comme préalable, la perpétuation des cadres étatiques. Pour notre propos, c'est là leur mérite particulier à ce stade.

70. Cahier des résolutions adoptées, congrès du CCA-NAC, juin 1991, section II Policy Resolutions : «Human Rights and the Constitution», résolutions $13,14,15$.

71. Philip Resnick, Toward a Canada-Quebec Union, Montréal et Kingston, McGill-Queen's University Press, 1991.

72. Daniel Drache et Roberto Perin, dir., Negotiating with a Sovereign Quebec, Toronto, James Lorimer and Co., 1992. 
Car la force et l'intérêt des analyses de la période waffle venaient, pensons-nous, de la volonté d'un traitement intégré des multiples traits, problèmes et développements historiques de l'État canadien, dans un esprit d'indépendance critique qui permettait de les aborder, notamment, en fonction de leurs rapports mutuels. La nature, à notre avis inachevée, de ce traitement a eu des conséquences théoriques lourdes de signification, ainsi que nous avons tenté de l'indiquer. En fin de compte, pourtant, ce qui semble le plus dramatique pour les sciences sociales, c'est qu'elle ait conduit à l'éclatement, ou a la disjonction, des lieux et axes d'appréciation des caractéristiques de l'État canadien.

Durant les années 1980 , les analyses critiques ont semblé en effet abandonner cette volonté d'articuler une perception et une théorie de l'État canadien qui auraient intégré les dimensions diverses de sa dynamique, telles que l'évolution des rapports constitutionnels, les questions nationales, les revendications des mouvements populaires, l'emprise économico-politique des États-Unis, etc. Tout s'est passé comme si chacun de ces champs pouvait être considéré séparément ou en ne tenant compte que de quelques-unes de ses relations avec l'ensemble du contenu.

II apparaît ainsi que les questions théoriques non lou mal) résolues de la première moitié des années 1970 avaient amené une telle fragmentation des champs d'analyse que les traitements unifiés de la réalité politique canadienne semblaient impossibles. Sur ce terrain, les sciences sociales sont théoriquement perdantes. À notre avis, la prémisse d'une nouvelle avancée significative de l'analyse politique progressiste sur le Québec et la constitution reposera, partiellement tout au moins, sur la volonté de revenir à un traitement intégré des multiples facettes de la réalité pancanadienne. Ce traitement devra se faire, cette fois, sur la base d'une réelle émancipation critique envers les institutions structurant l'État et le fédéralisme, et à partir des données et des besoins nationaux, sociaux et démocratiques, historiquement définis, des populations du territoire. 